\title{
Inteligibilidad acústica en español: una propuesta para su medición*
}

Acoustic intelligibility in Spanish: a proposal for its measurement

\author{
Claudia Rosas ${ }^{1}$, Jorge Sommerhoff ${ }^{2}$ \\ ${ }^{1}$ Universidad Austral de Chile, Instituto de Lingüística y Literatura, Apartado postal 567, Valdivia, \\ Chile, e-mail: claudiarosas@uach.cl \\ ${ }^{2}$ Universidad Austral de Chile, Instituto de Acústica, Apartado postal 567, Valdivia, Chile, \\ e-mail: jsommerh@uach.cl
}

El presente trabajo analiza el comportamiento de una lista de 1200 logatomos en español de estructura CVC (Consonante + Vocal + Consonante) con el objeto de elaborar varias listas de logatomos que permitan medir el porcentaje de articulación, como característica de calidad acústica de una sala para la voz hablada, y cuyos resultados sean comparables con las mediciones de STI. Como resultado se obtiene un corpus de 850 logatomos con los cuales se confeccionaron 17 listas de prueba de 50 logatomos cada una.

Palabras clave: inteligibilidad, mediciones, logatomos en español.

This work analyzes the behavior of a list of 1200 Spanish logatoms with a CVC (Consonant + Vowel + Consonant) structure in order to elaborate several lists of logatoms that allow for the measurement of articulation percentage, as characteristic of the acoustic quality of a room for the spoken word, and whose results are comparable with the measurements of STI. As a result, a corpus of 850 logatoms is obtained, with which 17 test lists of 50 logatoms were made.

Key words: intelligibility, measurements, Spanish logatoms.

\section{INTRODUCCIÓN}

Se define la inteligibilidad del habla como una medición de la efectividad para comprender la voz hablada ${ }^{1}$. Los procedimientos utilizados para ello se clasifican en dos tipos: subjetivos y objetivos. El método subjetivo consiste en contar los aciertos transcritos de un dictado de segmentos (sílabas, palabras o frases). El resultado de

\footnotetext{
* Este trabajo ha sido elaborado en el marco del proyecto de investigación Fondecyt 1070210 (Investigadora Responsable C. Rosas y Coinvestigador J. E. Sommerhoff). Para ello se han utilizado datos provenientes de un proyecto interno anterior Ref.: DID UACh S-2005-50 financiado por la Universidad Austral de Chile (Investigador Responsable J. E. Sommerhoff).

${ }^{1}$ Se ha utilizado "inteligibilidad del habla" en el sentido más tradicional y amplio que esta formulación tiene en el estudio de la voz: capacidad de comprensión del material lingüístico (Cf. Llisterri 1991: 118 y 119).
} 
este procedimiento, conocido como test de articulación ${ }^{2}$, depende fundamentalmente de dos variables: el corpus -la lista de segmentos- y la administración de la prueba o test. (Cf. Egan, J.P. 1948). El método objetivo, por su parte, utiliza máquinas, cuyos resultados se correlacionan con los obtenidos mediante el método subjetivo. Uno de los tests objetivos más utilizado corresponde al STI (Speech Transmition Index) desarrollado por Houtgast y Steeneken (1973). El STI es una técnica de medición que determina el grado de disminución que sufre la amplitud de la modulación de la señal sonora de prueba que se entrega a la sala, producto de la reverberación y ruido que existe en ella. Y esta diminución producida en el punto de recepción está directamente relacionada con la pérdida de inteligibilidad, cuyos resultados se distribuyen en una escala que va desde 0 (ininteligibilidad absoluta) a 1 (inteligibilidad absoluta).

A continuación, la siguiente figura 1 muestra la relación obtenida por Houtgast y Steeneken (1985) en el idioma inglés entre el sistema de prueba y el porcentaje de inteligibilidad medido utilizando un listado logatomos con estructura CVC (Consonante + Vocal + Consonante) y STI.

Figura 1

Relación entre prueba subjetiva de logatomos CVC en inglés y STI (Houtgast et al. 1985)

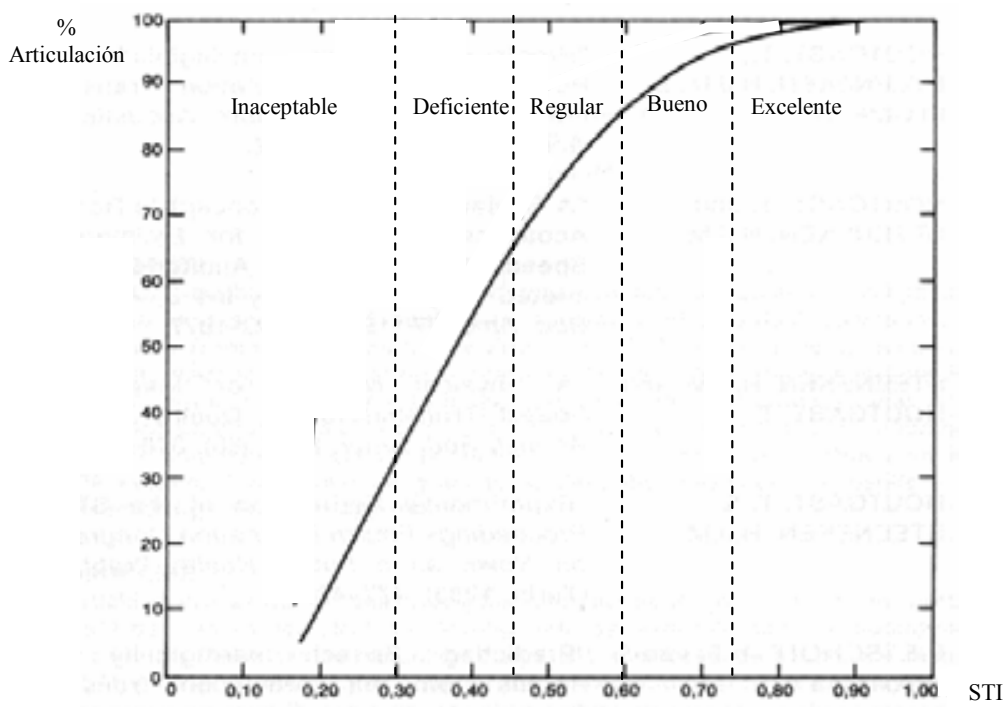

Dado que la prueba subjetiva de $\operatorname{logatomos}^{3}$ en el idioma español también es dependendiente del corpus utilizado (Cf. Sommerhoff y Rosas 2007: 224), en este

\footnotetext{
2 El uso extendido del término "prueba de articulación" permite aludir inequívocamente a los métodos subjetivos que utilizan en sus mediciones de inteligibilidad seres humanos que transcriben lo que oyen o lo que alguien "articula" frente a los denominados métodos objetivos que utilizan máquinas (Cf. Gavilán 1988: 244).

3 Palabras sin sentido, pero que formalmente se ajustan al sistema de una lengua cualquiera (por ejemplo: charchar, criso que son palabras inventadas en español) (Cf. Llisterri 1991: 71).
} 
trabajo se elaboró un corpus ad hoc con el objeto de obtener resultados para el español, en función del STI, semejantes a los que se obtienen de las listas del idioma inglés, establecidas por Houtgast y Steeneken.

\section{Metodología}

\subsection{EL CORPUS}

El corpus utilizado para medir la inteligibilidad está formado por 1200 logatomos de estructura CVC (Consonante + Vocal + Consonante) que incluye todas las combinaciones CVC fonéticamente posibles del español, representados en ortografía normal. Este corpus se presenta en cinco tablas, donde la tabla 1 corresponde a la vocal " $a$ " de la estructura CVC, la tabla 2 corresponde a la vocal "e" de la estructura CVC, y así sucesivamente hasta la vocal "u". Las filas de cada tabla se refieren a la primera consonante de CVC y las columnas a la segunda consonante de CVC. Los casilleros en blanco señalan combinaciones de consonantes no utilizadas, ya que coinciden fonéticamente con otras consonantes. Los casilleros con números y las sombras en algunos de ellos, corresponden a resultados que se explicarán más adelante.

Tabla 1

Combinaciones CVC con la vocal "a"

\begin{tabular}{|c|c|c|c|c|c|c|c|c|c|c|c|c|c|c|c|c|c|c|c|c|c|c|c|}
\hline $\mathbf{a}$ & B & $\mathrm{C}$ & $\mathrm{CH}$ & $\mathrm{D}$ & $\mathrm{F}$ & $\mathrm{G}$ & $\mathrm{H}$ & $\mathrm{J}$ & $\mathrm{K}$ & $\mathrm{L}$ & $M$ & $\mathrm{~N}$ & $\tilde{\mathrm{N}}$ & $\mathrm{P}$ & $\mathrm{Q}$ & $\mathrm{R}$ & $S$ & $\mathrm{~T}$ & $\mathrm{~V}$ & $\mathrm{~W}$ & $X$ & $\mathrm{Y}$ & $\mathrm{Z}$ \\
\hline B & 29 & & 96 & 21 & 25 & 57 & & 71 & 96 & 96 & 96 & 57 & & 46 & & 54 & 100 & 46 & & & 71 & & \\
\hline \multicolumn{24}{|l|}{ C } \\
\hline $\mathrm{CH}$ & 32 & & 79 & 39 & 50 & 25 & & 29 & 86 & 61 & 93 & 29 & & 39 & & 57 & 86 & 46 & & & 39 & & \\
\hline D & 54 & & 79 & 64 & 57 & 25 & & 18 & 32 & 75 & 25 & 57 & & 46 & & 7 & 86 & 43 & & & 75 & & \\
\hline $\mathrm{F}$ & 11 & & 79 & 11 & 25 & 11 & & 18 & 54 & 71 & 82 & 36 & & 36 & & 36 & 79 & 54 & & & 89 & & \\
\hline $\mathrm{G}$ & 39 & & 89 & 7 & 36 & 50 & & 39 & 75 & 39 & 82 & 57 & & 25 & & 29 & 96 & 54 & & & 61 & & \\
\hline \multicolumn{24}{|l|}{$\mathrm{H}$} \\
\hline $\mathrm{J}$ & 29 & & 82 & 14 & 4 & 36 & & 71 & 57 & 89 & 89 & 93 & & 39 & & 68 & 93 & 32 & & & 86 & & \\
\hline $\mathrm{K}$ & 54 & & 96 & 29 & 32 & 39 & & 29 & 93 & 86 & 96 & 68 & & 43 & & 61 & 100 & 68 & & & 89 & & \\
\hline $\mathrm{L}$ & 82 & & 79 & 21 & 43 & 36 & & 61 & 89 & 96 & 82 & 89 & & 50 & & 29 & 100 & 50 & & & 96 & & \\
\hline $\mathrm{M}$ & 36 & & 93 & 25 & 11 & 29 & & 86 & 89 & 100 & 100 & 96 & & 61 & & 82 & 96 & 75 & & & 68 & & \\
\hline $\mathrm{N}$ & 25 & & 86 & 36 & 11 & 36 & & 64 & 79 & 93 & 89 & 79 & & 68 & & 64 & 100 & 71 & & & 82 & & \\
\hline$\tilde{\mathrm{N}}$ & 54 & & 86 & 25 & 50 & 18 & & 18 & 93 & 68 & 96 & 86 & & 36 & & 25 & 96 & 57 & & & 79 & & \\
\hline $\mathrm{P}$ & 32 & & 79 & 25 & 43 & 50 & & 86 & 46 & 93 & 86 & 57 & & 64 & & 64 & 75 & 75 & & & 86 & & \\
\hline \multicolumn{24}{|l|}{$\mathrm{Q}$} \\
\hline $\mathrm{R}$ & 50 & & 96 & 7 & 25 & 29 & & 82 & 89 & 96 & 86 & 54 & & 82 & & 29 & 100 & 25 & & & 89 & & \\
\hline $\mathrm{S}$ & 46 & & 100 & 7 & 14 & 39 & & 36 & 93 & 96 & 86 & 64 & & 46 & & 68 & 100 & 43 & & & 93 & & \\
\hline $\mathrm{T}$ & 68 & & 96 & 18 & 21 & 46 & & 54 & 89 & 93 & 71 & 54 & & 36 & & 25 & 96 & 43 & & & 86 & & \\
\hline \multicolumn{24}{|l|}{$\mathrm{V}$} \\
\hline \multicolumn{24}{|l|}{$\mathrm{W}$} \\
\hline \multicolumn{24}{|l|}{$\mathrm{X}$} \\
\hline $\mathrm{Y}$ & 21 & & 79 & 25 & 0 & 21 & & 46 & 89 & 21 & 86 & 79 & & 79 & & 18 & 75 & 79 & & & 32 & & \\
\hline $\mathrm{Z}$ & & & & & & & & & & & & & & & & & & & & & & & \\
\hline
\end{tabular}


Tabla 2

Combinaciones CVC con la vocal "e"

\begin{tabular}{|c|c|c|c|c|c|c|c|c|c|c|c|c|c|c|c|c|c|c|c|c|c|c|c|}
\hline e & B & C & $\mathrm{CH}$ & $\mathrm{D}$ & $\mathrm{F}$ & G & $\mathrm{H}$ & $\mathrm{J}$ & $\mathrm{K}$ & L & M & $\mathrm{N}$ & \begin{tabular}{|l|}
$\tilde{N}$ \\
\end{tabular} & $P$ & $\mathrm{Q}$ & R & $\mathrm{S}$ & $\mathrm{T}$ & V & w & $\mathrm{X}$ & & \\
\hline B & 18 & & 46 & 25 & 18 & \begin{tabular}{|l|}
255 \\
\end{tabular} & & \begin{tabular}{|l|}
39 \\
\end{tabular} & 54 & \begin{tabular}{|l|}
82 \\
\end{tabular} & \begin{tabular}{|l|}
50 \\
\end{tabular} & 46 & & \begin{tabular}{|l|} 
\\
\end{tabular} & & 54 & 71 & 82 & & & 86 & & \\
\hline $\begin{array}{ll}\mathrm{C} \\
\end{array}$ & & & & & & & & & & & & & & & & & & & & & & & \\
\hline $\begin{array}{cc}\mathrm{CH} \\
\end{array}$ & 39 & & 79 & 7 & 75 & 11 & & 43 & 68 & \begin{tabular}{|l|} 
\\
\end{tabular} & \begin{tabular}{|l}
79 \\
\end{tabular} & 86 & & \begin{tabular}{|l|}
64 \\
\end{tabular} & & 25 & 86 & 54 & & & 68 & & \\
\hline $\mathrm{D}$ & 36 & & 93 & 21 & \begin{tabular}{|c|}
18 \\
\end{tabular} & \begin{tabular}{|l|}
14 \\
\end{tabular} & & 32 & 64 & 75 & \begin{tabular}{|l|}
96 \\
\end{tabular} & 32 & & 57 & & 82 & 79 & 39 & & & 57 & & \\
\hline $\mathrm{F}$ & 14 & & 64 & 7 & \begin{tabular}{|l|}
18 \\
\end{tabular} & 4 & & 21 & 57 & 75 & 54 & 21 & & 29 & & 43 & 93 & 68 & & & \begin{tabular}{l|}
61 \\
\end{tabular} & & \\
\hline$\overline{\mathrm{GU}}$ & 54 & & 75 & 29 & \begin{tabular}{|l|}
7 \\
\end{tabular} & 21 & & 36 & 82 & 43 & 61 & 82 & & 36 & & 39 & 57 & 61 & & & 89 & & \\
\hline $\mathrm{H}$ & & & & & & & & & & & & & & & & & & & & & & & \\
\hline $\mathrm{J}$ & 29 & & 89 & 18 & 57 & 11 & & \begin{tabular}{|l|}
71 \\
\end{tabular} & 43 & 25 & 82 & 68 & & \begin{tabular}{|l|}
61 \\
\end{tabular} & & 82 & 93 & 50 & & & 75 & & \\
\hline $\mathrm{K}$ & 32 & & 82 & 28 & \begin{tabular}{|l|}
39 \\
\end{tabular} & \begin{tabular}{|l|}
36 \\
\end{tabular} & & \begin{tabular}{|l|}
36 \\
\end{tabular} & 79 & \begin{tabular}{|l|}
82 \\
\end{tabular} & \begin{tabular}{|l}
68 \\
\end{tabular} & 68 & & \begin{tabular}{|l|} 
\\
\end{tabular} & & 64 & 89 & 64 & & & 75 & & \\
\hline $\begin{array}{ll}\mathrm{L} \\
\end{array}$ & 57 & & 100 & 46 & 57 & \begin{tabular}{|l|}
32 \\
\end{tabular} & & \begin{tabular}{|l|}
57 \\
\end{tabular} & $\begin{array}{l}96 \\
\end{array}$ & $\begin{array}{l}79 \\
\end{array}$ & 100 & 82 & & 75 & & 71 & 89 & 68 & & & 93 & & \\
\hline $\mathrm{M}$ & 11 & & 100 & 39 & \begin{tabular}{|l|}
14 \\
\end{tabular} & \begin{tabular}{|l|}
36 \\
\end{tabular} & & 75 & 96 & \begin{tabular}{|l|}
75 \\
\end{tabular} & \begin{tabular}{|l|}
93 \\
\end{tabular} & 89 & & \begin{tabular}{|l|}
64 \\
\end{tabular} & & 89 & 89 & 71 & & & 96 & & \\
\hline $\mathrm{N}$ & 46 & & 50 & 18 & 57 & \begin{tabular}{|l|}
29 \\
\end{tabular} & & \begin{tabular}{|l|}
50 \\
\end{tabular} & 86 & 54 & \begin{tabular}{|l}
96 \\
\end{tabular} & 29 & & \begin{tabular}{|l|}
82 \\
\end{tabular} & & 61 & 96 & 29 & & & 89 & & \\
\hline$\tilde{\mathrm{N}}$ & 11 & & 89 & 14 & 21 & 29 & & 54 & 86 & 57 & 82 & 39 & & 36 & & 46 & 75 & 32 & & & 89 & & \\
\hline $\mathrm{P}$ & 46 & & 96 & 18 & \begin{tabular}{|l|}
18 \\
\end{tabular} & 25 & & 64 & 93 & 93 & \begin{tabular}{|l|}
79 \\
\end{tabular} & 93 & & 64 & & 75 & 96 & 89 & & & 64 & & \\
\hline$Q$ & & & & & & & & & & & & & & & & & & & & & & & \\
\hline $\mathrm{R}$ & 21 & & 100 & 36 & 25 & 36 & & 18 & 79 & \begin{tabular}{|l|}
68 \\
\end{tabular} & \begin{tabular}{|l}
79 \\
\end{tabular} & 54 & & \begin{tabular}{|l|} 
\\
\end{tabular} & & 50 & 96 & 39 & & & \begin{tabular}{l|}
61 \\
6
\end{tabular} & & \\
\hline $\mathrm{s}$ & 64 & & 93 & 14 & \begin{tabular}{|l|}
36 \\
\end{tabular} & 21 & & 50 & 89 & \begin{tabular}{|l|}
89 \\
\end{tabular} & \begin{tabular}{|l|} 
\\
\end{tabular} & 93 & & 54 & & 75 & 93 & 39 & & & 89 & & \\
\hline $\mathrm{T}$ & 25 & & 71 & 25 & \begin{tabular}{|l|}
11 \\
\end{tabular} & \begin{tabular}{|l|}
18 \\
\end{tabular} & & 75 & 57 & \begin{tabular}{|l|}
79 \\
\end{tabular} & 86 & 57 & & 32 & & 79 & 75 & 29 & & & \begin{tabular}{l|}
71 \\
\end{tabular} & & \\
\hline $\mathrm{v}$ & & & & & & & & & & & & & & & & & & & & & & & \\
\hline $\mathrm{w}$ & & & & & & & & & & & & & & & & & & & & & & & \\
\hline$x$ & & & & & & & & & & & & & & & & & & & & & & & \\
\hline $\begin{array}{l}\mathrm{Y} \\
\end{array}$ & 29 & & 79 & 7 & \begin{tabular}{|l|}
68 \\
\end{tabular} & 36 & & \begin{tabular}{|l|}
68 \\
\end{tabular} & 79 & 32 & 82 & 75 & & 50 & & 54 & 100 & 71 & & & 64 & & \\
\hline & & & & & & & & & & & & & & & & & & & & & & & \\
\hline
\end{tabular}

Tabla 3

Combinaciones CVC con la vocal " $\mathrm{i}$ "

\begin{tabular}{|c|c|c|c|c|c|c|c|c|c|c|c|c|c|c|c|c|c|c|c|c|c|c|c|}
\hline i & B & C & $\mathrm{CH}$ & D & $\mathrm{F}$ & $\mathrm{G}$ & $\mathrm{H}$ & $\mathrm{J}$ & $\mathrm{K}$ & L & M & $\mathrm{N}$ & $\tilde{\mathrm{N}}$ & $P$ & Q & $\mathrm{R}$ & $\underline{s}$ & $\mathrm{~T}$ & $\mathrm{~V}$ & $\mathrm{~W}$ & $X$ & $\mathrm{Y}$ & Z \\
\hline B & 25 & & \begin{tabular}{|l}
89 \\
\end{tabular} & 14 & 39 & 29 & & \begin{tabular}{|l}
79 \\
\end{tabular} & 68 & 79 & \begin{tabular}{|l|}
71 \\
\end{tabular} & \begin{tabular}{|l|l}
89 \\
\end{tabular} & & 36 & & 36 & \begin{tabular}{|l|l}
100 \\
\end{tabular} & \begin{tabular}{|l|l}
39 \\
\end{tabular} & & & 46 & & \\
\hline $\mathrm{C}$ & & & & & & & & & & & & & & & & & & & & & & & \\
\hline $\mathrm{CH}$ & 39 & & 75 & 18 & \begin{tabular}{|l|}
61 \\
\end{tabular} & 25 & & 50 & 32 & 71 & \begin{tabular}{|l}
68 \\
\end{tabular} & 89 & & 68 & & 86 & \begin{tabular}{|l|l}
96 \\
\end{tabular} & 36 & & & 93 & & \\
\hline $\mathrm{D}$ & 0 & & \begin{tabular}{|l}
93 \\
\end{tabular} & 11 & 54 & \begin{tabular}{|l|}
46 \\
\end{tabular} & & 64 & \begin{tabular}{|l}
79 \\
\end{tabular} & 82 & \begin{tabular}{|l}
57 \\
\end{tabular} & \begin{tabular}{|l|l|} 
\\
\end{tabular} & & \begin{tabular}{|l}
68 \\
\end{tabular} & & \begin{tabular}{|l|}
46 \\
\end{tabular} & \begin{tabular}{|l|}
71 \\
\end{tabular} & 18 & & & 96 & & \\
\hline $\mathrm{F}$ & 32 & & 100 & 11 & 68 & 29 & & 32 & 71 & 93 & 54 & 96 & & 21 & & 64 & 36 & 64 & & & 64 & & \\
\hline $\mathrm{GU}$ & 25 & & \begin{tabular}{|l}
79 \\
\end{tabular} & 25 & 36 & 25 & & \begin{tabular}{|l|}
21 \\
\end{tabular} & \begin{tabular}{|l|}
61 \\
\end{tabular} & 14 & \begin{tabular}{|l}
68 \\
\end{tabular} & \begin{tabular}{|l|}
64 \\
\end{tabular} & & \begin{tabular}{|l}
75 \\
\end{tabular} & & \begin{tabular}{|l}
54 \\
\end{tabular} & 57 & \begin{tabular}{|l|} 
\\
\end{tabular} & & & 71 & & \\
\hline $\mathrm{H}$ & & & & & & & & & & & & & & & & & & & & & & & \\
\hline $\mathrm{J}$ & 43 & & \begin{tabular}{|l}
82 \\
\end{tabular} & 0 & 68 & \begin{tabular}{|l}
7 \\
\end{tabular} & & \begin{tabular}{|l}
68 \\
\end{tabular} & \begin{tabular}{|l}
82 \\
\end{tabular} & 29 & \begin{tabular}{|l|}
86 \\
\end{tabular} & \begin{tabular}{|l|}
54 \\
\end{tabular} & & \begin{tabular}{|l}
68 \\
\end{tabular} & & \begin{tabular}{|l|}
43 \\
\end{tabular} & 75 & 77 & & & \begin{tabular}{|l}
79 \\
\end{tabular} & & \\
\hline $\mathrm{K}$ & 36 & & 89 & 18 & 79 & 29 & & 39 & \begin{tabular}{|l|}
71 \\
\end{tabular} & 86 & 75 & 68 & & \begin{tabular}{|l|}
39 \\
\end{tabular} & & 64 & 82 & 29 & & & 71 & & \\
\hline $\mathrm{L}$ & 71 & & 100 & 7 & 39 & 32 & & \begin{tabular}{|l}
68 \\
\end{tabular} & \begin{tabular}{|l}
75 \\
\end{tabular} & 50 & \begin{tabular}{|l}
89 \\
\end{tabular} & \begin{tabular}{|l|}
64 \\
\end{tabular} & & \begin{tabular}{|l|}
61 \\
\end{tabular} & & \begin{tabular}{|l|}
21 \\
\end{tabular} & 100 & \begin{tabular}{|l|}
61 \\
\end{tabular} & & & 86 & & \\
\hline $\mathrm{M}$ & 11 & & 54 & 18 & 54 & 25 & & 71 & \begin{tabular}{|l|}
71 \\
\end{tabular} & 57 & 75 & 64 & & 57 & & 96 & 82 & 68 & & & 93 & & \\
\hline $\mathrm{N}$ & 21 & & \begin{tabular}{|l|}
79 \\
\end{tabular} & 21 & 25 & 46 & & \begin{tabular}{|l|}
68 \\
\end{tabular} & \begin{tabular}{|l|l} 
\\
\end{tabular} & 54 & \begin{tabular}{|l}
71 \\
\end{tabular} & \begin{tabular}{|l}
61 \\
\end{tabular} & & \begin{tabular}{|l}
11 \\
\end{tabular} & & \begin{tabular}{|l}
79 \\
\end{tabular} & 79 & 50 & & & 79 & & \\
\hline$\tilde{\mathrm{N}}$ & 39 & & 96 & 18 & 43 & 25 & & 61 & 68 & 39 & \begin{tabular}{|l}
93 \\
\end{tabular} & 39 & & \begin{tabular}{|l}
89 \\
\end{tabular} & & 61 & \begin{tabular}{|l|}
93 \\
\end{tabular} & 18 & & & \begin{tabular}{|l}
89 \\
\end{tabular} & & \\
\hline $\mathrm{P}$ & 36 & & \begin{tabular}{|l|}
96 \\
\end{tabular} & 4 & \begin{tabular}{|l}
68 \\
\end{tabular} & 32 & & \begin{tabular}{|l|} 
\\
\end{tabular} & \begin{tabular}{|l}
89 \\
\end{tabular} & 82 & \begin{tabular}{|l|l}
64 \\
\end{tabular} & \begin{tabular}{|l}
71 \\
\end{tabular} & & \begin{tabular}{|l}
68 \\
\end{tabular} & & \begin{tabular}{|l|}
86 \\
\end{tabular} & \begin{tabular}{|l}
86 \\
\end{tabular} & 64 & & & 82 & & \\
\hline$Q$ & & & & & & & & & & & & & & & & & & & & & & & \\
\hline$R$ & 18 & & 82 & 14 & 18 & 39 & & 89 & 86 & \begin{tabular}{|l|}
46 \\
\end{tabular} & 57 & 57 & & 54 & & 46 & \begin{tabular}{|l|l}
100 \\
\end{tabular} & 57 & & & 93 & & \\
\hline $\mathrm{s}$ & 14 & & 96 & 25 & 82 & 25 & & 18 & \begin{tabular}{|l|}
96 \\
\end{tabular} & 89 & 86 & 82 & & 54 & & \begin{tabular}{|l}
89 \\
\end{tabular} & 86 & 61 & & & 89 & & \\
\hline $\mathrm{T}$ & 39 & & \begin{tabular}{|l}
68 \\
\end{tabular} & 39 & 79 & \begin{tabular}{|l|}
29 \\
\end{tabular} & & \begin{tabular}{|l|}
50 \\
\end{tabular} & \begin{tabular}{|l|l}
64 \\
\end{tabular} & 54 & \begin{tabular}{|l|}
96 \\
\end{tabular} & \begin{tabular}{|l|l} 
\\
\end{tabular} & & \begin{tabular}{|l}
54 \\
\end{tabular} & & \begin{tabular}{|l}
93 \\
\end{tabular} & 64. & \begin{tabular}{|l|l} 
\\
\end{tabular} & & & \begin{tabular}{|l}
93 \\
\end{tabular} & & \\
\hline $\mathrm{v}$ & & & & & & & & & & & & & & & & & & & & & & & \\
\hline $\mathrm{w}$ & & & & & & & & & & & & & & & & & & & & & & & \\
\hline $\mathrm{x}$ & & & & & & & & & & & & & & & & & & & & & & & \\
\hline $\mathrm{Y}$ & 50 & & 86 & 11 & 79 & \begin{tabular}{|l|} 
\\
\end{tabular} & & 25 & 46 & 39 & 50 & 68 & & 57 & & 57 & 86 & 25 & & & 71 & & \\
\hline $\mathrm{z}$ & & & & & & & & & & & & & & & & & & & & & & & \\
\hline
\end{tabular}


Tabla 4

Combinaciones CVC con la vocal "o"

\begin{tabular}{|c|c|c|c|c|c|c|c|c|c|c|c|c|c|c|c|c|c|c|c|c|c|c|c|}
\hline $\mathbf{0}$ & B & $\mathrm{C}$ & $\mathrm{CH}$ & $\mathrm{D}$ & $\mathrm{F}$ & $\mathrm{G}$ & $\mathrm{H}$ & $\mathrm{J}$ & $\mathrm{K}$ & $\mathrm{L}$ & $\mathrm{M}$ & $\mathrm{N}$ & $\tilde{\mathrm{N}}$ & $\mathrm{P}$ & $\mathrm{Q}$ & $\mathrm{R}$ & S & $\mathrm{T}$ & $\mathrm{V}$ & $\mathrm{W}$ & $\mathrm{X}$ & $\mathrm{Y}$ & $\mathrm{Z}$ \\
\hline $\mathrm{B}$ & 36 & & 89 & 18 & 64 & 18 & & 86 & 96 & 93 & 79 & 46 & & 54 & & 96 & 93 & 89 & & & 50 & & \\
\hline \multicolumn{24}{|l|}{$\mathrm{C}$} \\
\hline $\mathrm{CH}$ & 29 & & 79 & 25 & 36 & 14 & & 43 & 79 & 82 & 71 & 21 & & 32 & & 86 & 100 & 89 & & & 82 & & \\
\hline D & 32 & & 89 & 14 & 86 & 46 & & 46 & 75 & 57 & 93 & 57 & & 18 & & 61 & 93 & 93 & & & 68 & & \\
\hline $\mathrm{F}$ & 7 & & 50 & 29 & 25 & 43 & & 43 & 71 & 54 & 68 & 82 & & 36 & & 75 & 71 & 79 & & & 61 & & \\
\hline $\mathrm{G}$ & 43 & & 89 & 32 & 71 & 50 & & 61 & 46 & 100 & 68 & 32 & & 54 & & 71 & 79 & 79 & & & 25 & & \\
\hline \multicolumn{24}{|l|}{$\mathrm{H}$} \\
\hline $\mathrm{J}$ & 11 & & 89 & 0 & 50 & 14 & & 64 & 54 & 71 & 89 & 89 & & 71 & & 86 & 93 & 68 & & & 89 & & \\
\hline $\mathrm{K}$ & 18 & & 100 & 18 & 86 & 36 & & 61 & 75 & 89 & 93 & 89 & & 36 & & 89 & 96 & 61 & & & 89 & & \\
\hline $\mathrm{L}$ & 36 & & 93 & 29 & 71 & 29 & & 46 & 71 & 79 & 64 & 64 & & 64 & & 61 & 96 & 100 & & & 82 & & \\
\hline M & 32 & & 82 & 11 & 11 & 25 & & 50 & 82 & 96 & 32 & 75 & & 79 & & 96 & 96 & 89 & & & 89 & & \\
\hline $\mathrm{N}$ & 36 & & 75 & 21 & 32 & 7 & & 79 & 93 & 61 & 79 & 82 & & 39 & & 93 & 96 & 93 & & & 82 & & \\
\hline$\tilde{\mathrm{N}}$ & 29 & & 89 & 21 & 57 & 18 & & 89 & 96 & 46 & 79 & 71 & & 64 & & 75 & 96 & 89 & & & 68 & & \\
\hline $\mathrm{P}$ & 25 & & 89 & 36 & 64 & 36 & & 46 & 75 & 96 & 68 & 54 & & 57 & & 89 & 86 & 89 & & & 89 & & \\
\hline \multicolumn{24}{|l|}{$\mathrm{Q}$} \\
\hline $\mathrm{R}$ & 21 & & 93 & 11 & 57 & 46 & & 54 & 82 & 54 & 82 & 61 & & 14 & & 25 & 100 & 64 & & & 89 & & \\
\hline $\mathrm{S}$ & 39 & & 96 & 18 & 79 & 25 & & 14 & 36 & 89 & 86 & 57 & & 61 & & 93 & 100 & 61 & & & 100 & & \\
\hline $\mathrm{T}$ & 21 & & 93 & 21 & 32 & 25 & & 75 & 89 & 93 & 96 & 68 & & 32 & & 100 & 96 & 68 & & & 82 & & \\
\hline \multicolumn{24}{|l|}{ V } \\
\hline \multicolumn{24}{|l|}{ W } \\
\hline \multicolumn{24}{|l|}{$\mathrm{X}$} \\
\hline $\mathrm{Y}$ & 43 & & 100 & 25 & 68 & 21 & & 57 & 86 & 71 & 82 & 79 & & 36 & & 68 & 96 & 82 & & & 86 & & \\
\hline $\mathrm{Z}$ & & & & & & & & & & & & & & & & & & & & & & & \\
\hline
\end{tabular}

Tabla 5

Combinaciones CVC con la vocal "u"

\begin{tabular}{|c|c|c|c|c|c|c|c|c|c|c|c|c|c|c|c|c|c|c|c|c|c|c|c|}
\hline $\mathbf{u}$ & B & $\mathrm{C}$ & $\mathrm{CH}$ & $\mathrm{D}$ & $\mathrm{F}$ & $\mathrm{G}$ & $\mathrm{H}$ & $\mathrm{J}$ & $\mathrm{K}$ & $\mathrm{L}$ & M & $\mathrm{N}$ & $\tilde{\mathrm{N}}$ & $\mathrm{P}$ & Q & $\mathrm{R}$ & $\mathrm{S}$ & $\mathrm{T}$ & $\mathrm{V}$ & W & $\mathrm{X}$ & $\mathrm{Y}$ & $\mathrm{Z}$ \\
\hline B & 25 & & 96 & 25 & 25 & 46 & & 57 & 21 & 82 & 61 & 50 & & 75 & & 86 & 100 & 93 & & & 86 & & \\
\hline \multicolumn{24}{|l|}{$\mathrm{C}$} \\
\hline $\mathrm{CH}$ & 14 & & 79 & 36 & 71 & 36 & & 46 & 93 & 93 & 68 & 39 & & 43 & & 61 & 86 & 96 & & & 75 & & \\
\hline $\mathrm{D}$ & 25 & & 82 & 25 & 79 & 4 & & 21 & 68 & 29 & 75 & 39 & & 36 & & 75 & 75 & 50 & & & 71 & & \\
\hline $\mathrm{F}$ & 11 & & 79 & 11 & 32 & 25 & & 25 & 64 & 93 & 61 & 32 & & 71 & & 75 & 71 & 93 & & & 57 & & \\
\hline $\mathrm{G}$ & 14 & & 46 & 25 & 50 & 21 & & 21 & 71 & 71 & 75 & 79 & & 64 & & 82 & 82 & 100 & & & 57 & & \\
\hline \multicolumn{24}{|l|}{$\mathrm{H}$} \\
\hline $\mathrm{J}$ & 29 & & 79 & 43 & 50 & 29 & & 71 & 64 & 82 & 64 & 57 & & 29 & & 39 & 68 & 96 & & & 86 & & \\
\hline $\mathrm{K}$ & 14 & & 93 & 14 & 57 & 11 & & 79 & 100 & 96 & 39 & 75 & & 54 & & 93 & 89 & 96 & & & 64 & & \\
\hline $\mathrm{L}$ & 29 & & 96 & 46 & 54 & 29 & & 64 & 93 & 89 & 89 & 57 & & 71 & & 46 & 100 & 86 & & & 100 & & \\
\hline $\mathrm{M}$ & 25 & & 96 & 4 & 64 & 39 & & 50 & 93 & 46 & 61 & 82 & & 43 & & 96 & 93 & 89 & & & 96 & & \\
\hline $\mathrm{N}$ & 14 & & 82 & 4 & 61 & 7 & & 36 & 96 & 82 & 82 & 68 & & 54 & & 54 & 79 & 82 & & & 86 & & \\
\hline$\tilde{\mathrm{N}}$ & 32 & & 75 & 21 & 79 & 4 & & 43 & 79 & 82 & 100 & 57 & & 54 & & 64 & 89 & 68 & & & 64 & & \\
\hline $\mathrm{P}$ & 57 & & 93 & 18 & 86 & 54 & & 61 & 89 & 89 & 89 & 93 & & 14 & & 61 & 93 & 82 & & & 89 & & \\
\hline \multicolumn{24}{|l|}{$\mathrm{Q}$} \\
\hline $\mathrm{R}$ & 25 & & 96 & 4 & 71 & 7 & & 46 & 79 & 96 & 46 & 43 & & 43 & & 43 & 96 & 100 & & & 93 & & \\
\hline $\mathrm{S}$ & 32 & & 93 & 36 & 36 & 18 & & 64 & 82 & 86 & 79 & 54 & & 54 & & 93 & 96 & 61 & & & 93 & & \\
\hline $\mathrm{T}$ & 64 & & 93 & 14 & 96 & 50 & & 82 & 89 & 100 & 61 & 68 & & 54 & & 86 & 93 & 57 & & & 82 & & \\
\hline \multicolumn{23}{|l|}{ V } & \\
\hline \multicolumn{24}{|l|}{$\mathrm{W}$} \\
\hline \multicolumn{24}{|l|}{$\mathrm{X}$} \\
\hline $\mathrm{Y}$ & 21 & & 82 & 0 & 32 & 32 & & 57 & 68 & 93 & 68 & 36 & & 61 & & 54 & 79 & 96 & & & 89 & & \\
\hline $\mathrm{Z}$ & & & & & & & & & & & & & & & & & & & & & & & \\
\hline
\end{tabular}




\subsection{MATERIALES Y METODOS}

La metodología general seguida para la realización del objetivo propuesto consideró tres aspectos: constitución del corpus inicial; administración de la prueba, y obtención del corpus final. Cada uno, a su vez, consideró una serie de tareas específicas como se describe a continuación.

\subsubsection{Constitución del corpus inicial}

2.2.1.1. Formación del corpus ad hoc: se construyó un corpus de 1200 logatomos de estructura CVC (Consonante + Vocal + Consonante) que incluye todas las combinaciones posibles del español.

2.2.1.2. Distribución del corpus: el corpus inicial de 1200 logatomos se dividió en doce listas de cien unidades cada una.

2.2.1.3. Digitalización de las listas: las listas ordenadas fueron grabadas en una sala anecoica con una separación de 1,5 s entre cada logatomo, a las que se agregaron frases introductorias ("pongan atención”, "ahora escuchen", "escuchen a continuación”, etc). Finalmente, se digitalizó la potencia acústica, ajustándola a la de la voz de una persona normal.

\subsubsection{Administración de la prueba}

2.2.2.1. Selección de los auditores: se utilizaron 30 estudiantes universitarios, de ambos sexos, sanos auditivamente y elegidos al azar, los cuales se distribuyeron en seis grupos de cinco individuos cada uno. Se les explicó el procedimiento y propósito de la tarea, como se detalla más adelante.

2.2.2.2. Selección de los recintos: se eligieron salas con un STI entre 0.38 y 0.7 , rango de valores donde más claramente se manifiestan las diferencias de la relación entre STI y \% de articulación, del corpus utlizado. Los recintos ad hoc utilizados se encuentran en Campus Miraflores ${ }^{4}$ y en el Campus Teja de la Universidad Austral de Chile.

2.2.2.3. Selección de los instrumentos de medición: para medir el STI se utilizó el medidor NTI Acoustilyzer junto con la fuente sonora NTI Talk Box que imita la voz humana (tanto en su potencia acústica como en su directividad) y que, gracias a su tarjeta Compact Flash, permite el almacenamiento de las señales que emite, en nuestro caso: STI y corpus de logatomos.

2.2.2.4. Aplicación de la prueba: los auditores se distribuyeron en distintos puntos de recepción de las salas desde donde escribieron las palabras escuchadas, en plantillas adaptadas al propósito. En esos mismos lugares se midió el STI de acuerdo al montaje que se muestra en la figura 2.

4 Posteriormente demolidos en febrero de 2007. 
Figura 2

Montaje para las mediciones en sala de prueba.

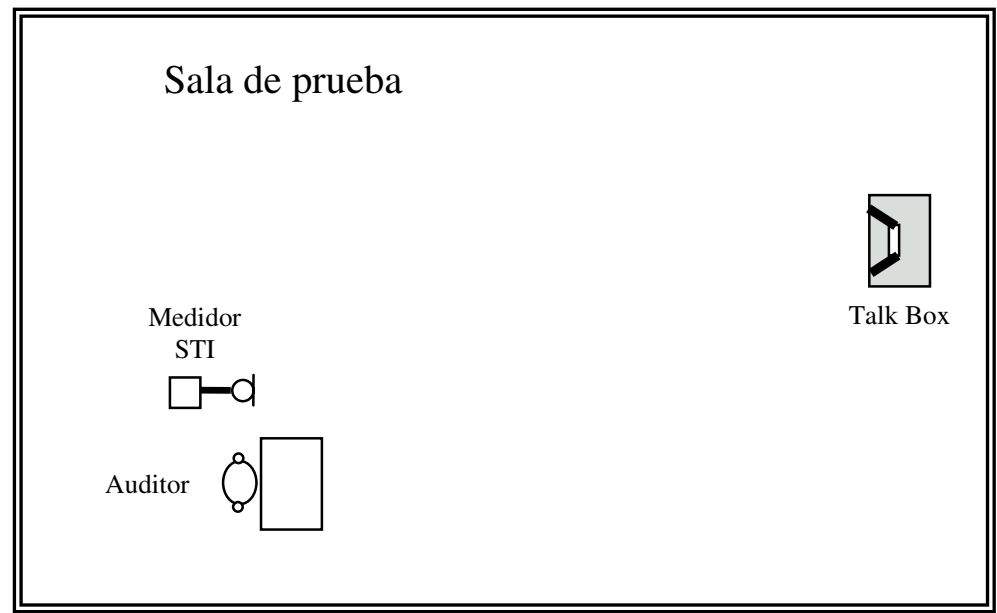

\subsubsection{Obtención del corpus final}

2.2.3.1. Análisis comparativo de las mediciones: los porcentajes de inteligibilidad obtenidos en la prueba de articulación fueron pareados con los resultados del STI. 2.2.3.2. Afinación del corpus final: se fueron eliminando sucesivamente los logatomos más difíciles de escuchar hasta lograr un nivel semejante al obtenido por Houtgast y Steeneken en la curva de relación entre STI y \% de articulación, con un total de 850 logatomos.

2.2.3.3. Presentación del corpus: el corpus final obtenido se divide en 17 listas de 50 logatomos balanceadas en cuanto a criterios de composición fonética CVC y grado de dificultad observado.

\section{Resultados}

En los casilleros de tablas 1, 2, 3, 4 y 5 se indica el porcentaje global de aciertos, considerando la totalidad de las pruebas realizadas. Este resultado es indicativo de la dificultad relativa que existe entre cada logatomo CVC de ser escuchado con claridad en distintas condiciones acústicas. Así, por ejemplo, el logatomo JAB (con un 29\% de aciertos) es más difícil de escucharlo en condiciones acústicamente adversas que el logatomo LUM (con un $89 \%$ de aciertos).

Los errores observados que se cometen al escuchar los logatomos pueden ser de cinco tipos, los cuales se identifican en la tabla 6. 
Tabla 6

Clasificación de los errores

\begin{tabular}{|l|l|}
\hline TIPO DE ERROR & \multicolumn{1}{|c|}{ DESCRIPCION } \\
\hline Error 1 & Escuchar incorrectamente la primera consonante de CVC \\
\hline Error 2 & Escuchar incorrectamente la vocal de CVC \\
\hline Error 3 & Escuchar incorrectamente la segunda consonante de CVC \\
\hline Error 4 & Escuchar incorrectamente las dos consonantes de CVC \\
\hline Error 5 & Escuchar incorrectamente las dos consonantes y vocal de CVC \\
\hline
\end{tabular}

De acuerdo a esta clasificación, el porcentaje de error cometido en cada categoría de distribución del corpus seleccionado se muestra en la tabla 7. En esta tabla se evidencia que el gran porcentaje de los errores ocurre al escuchar las consonantes $\mathrm{y}$, principalmente, al escuchar la segunda consonante del logatomo con estructura $\mathrm{CVC}^{5}$.

Tabla 7

Distribución de los tipos de errores en porcentaje de ocurrencia

\begin{tabular}{|c|c|c|c|c|}
\hline Error 1 & Error 2 & Error 3 & Error 4 & Error 5 \\
\hline $21 \%$ & $1,9 \%$ & $61 \%$ & $9 \%$ & $7 \%$ \\
\hline
\end{tabular}

Este resultado es coherente con lo publicado por Peutz (1971: 23-27) donde se establece que la pérdida de articulación de las consonantes está relacionada en una forma simple con la acústica de la sala y la relación señal-ruido, y por lo tanto, puede ser utilizada como una medida confiable de la inteligibilidad del habla. También, está claro que el mayor número de errores ocurre al escuchar la segunda consonante de CVC puesto que está precedida por la primera consonante y vocal, que se mantienen en la sala debido a la reverberancia del recinto, enmascarándola más o menos según su tiempo de reverberación. De esto se concluye que las listas deben estar balanceadas fonéticamente con la segunda consonante.

La figura 3 muestra el resultado de la relación de entre el \% de inteligibilidad y el STI medidos para el conjunto de los 1200 logatomos CVC, para el conjunto de 1000 logatomos de mayor acierto, para el conjunto 950 logatomos de mayor acierto, para el conjunto 900 logatomos de mayor acierto y para el conjunto 850 logatomos de mayor acierto, todos ellos en comparación con la curva de Houtgast y Steeneken.

Dado que la curva de resultado del conjunto de los 1200 logatomos está bajo la curva de Houtgast y Steeneken, se procedió a eliminar paulatinamente los logatomos con menor acierto, hasta lograr una curva con una altura semejante, como se muestra en la figura 3.

\footnotetext{
5 Sobre la incidencia de las características articulatorias en el porcentaje de inteligibilidad ver: ¿Existe la inteligibilidad absoluta? fonemas críticos en español (Sommerhoff y Rosas, en preparación).
} 
Figura 3

Relación de entre el \% de inteligibilidad y el STI para distintos conjuntos de logatomos

CVC de mayor acierto en comparación con la curva de Houtgast y Steeneken

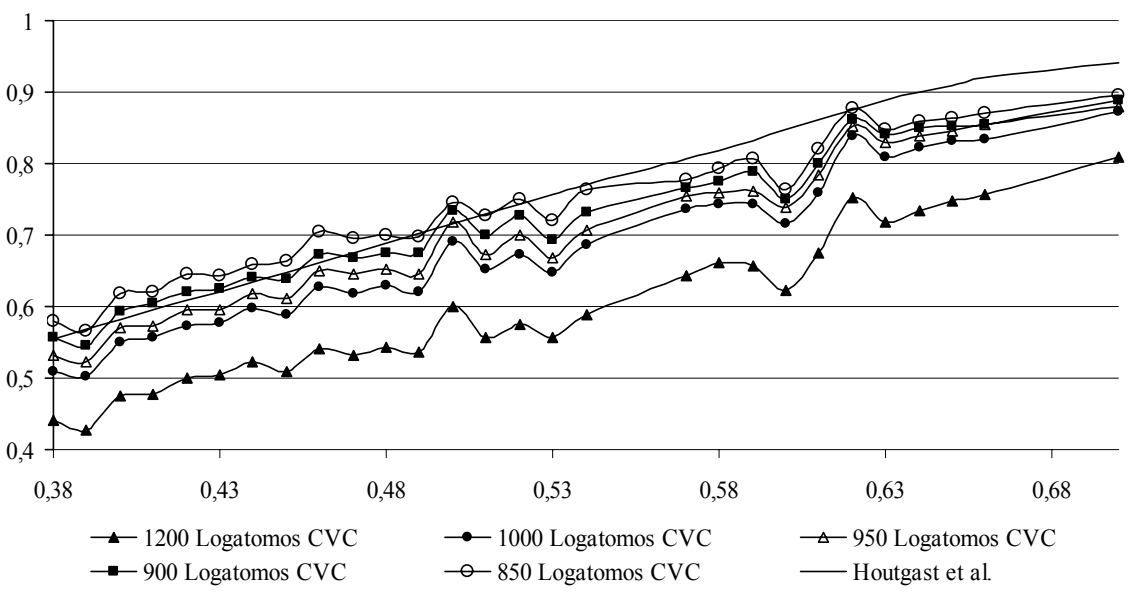

Así, se determinó que la curva que considera a los 850 logatomos con mayor acierto es la más cercana a la de Houtgast y Steeneken como se muestra en la figura 4.

Figura 4

Curva lineal de mejor ajuste de las curvas de 1200 CVC y 850 CVC en comparación con la curva de Houtgast y Steeneken

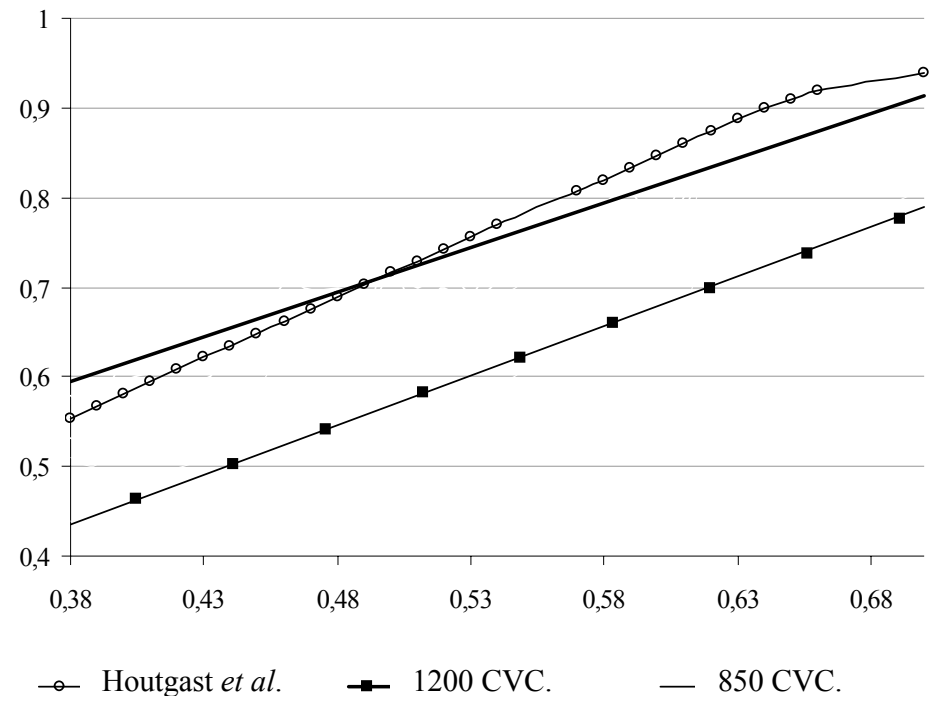

A continuación, la tabla 8 muestra la distribución balanceada de las segundas consonantes de cada lista. Se observa que en cada fila (consonante), la diferencia de ocurrencia de cada consonante entre las distintas columnas (listas) nunca es mayor a 1 , excepto en la fila de la consonante $\mathrm{F}$ donde en un solo caso alcanza la diferencia de 2. 
Tabla 8

Ocurrencia de la segunda consonante en cada lista

\begin{tabular}{|c|c|c|c|c|c|c|c|c|c|c|c|c|c|c|c|c|c|c|}
\hline \multirow{2}{*}{$\begin{array}{c}2^{\mathrm{da}} \\
\text { Consonante }\end{array}$} & \multicolumn{17}{|c|}{ LISTAS } & \multirow{2}{*}{ Total } \\
\hline & 1 & 2 & 3 & 4 & 5 & 6 & 7 & 8 & 9 & 10 & 11 & 12 & 13 & 14 & 15 & 16 & 17 & \\
\hline B & 1 & 1 & 1 & 1 & 1 & 1 & 1 & 1 & 2 & 2 & 1 & 1 & 1 & 1 & 1 & 1 & 1 & 19 \\
\hline $\mathrm{CH}$ & 5 & 5 & 4 & 5 & 5 & 4 & 4 & 5 & 5 & 5 & 5 & 5 & 5 & 5 & 5 & 4 & 4 & 80 \\
\hline D & 1 & 1 & 0 & 0 & 0 & 0 & 0 & 0 & 0 & 0 & 0 & 0 & 0 & 0 & 0 & 1 & 1 & 4 \\
\hline $\mathrm{F}$ & 3 & 2 & 3 & 3 & 3 & 3 & 3 & 4 & 3 & 3 & 2 & 2 & 2 & 2 & 2 & 2 & 2 & 44 \\
\hline $\mathrm{G}$ & 1 & 1 & 1 & 1 & 1 & 1 & 1 & 0 & 0 & 0 & 1 & 1 & 1 & 1 & 1 & 1 & 1 & 14 \\
\hline $\mathrm{J}$ & 3 & 3 & 3 & 3 & 4 & 4 & 3 & 3 & 4 & 4 & 4 & 4 & 3 & 3 & 3 & 3 & 3 & 57 \\
\hline $\mathrm{K}$ & 4 & 5 & 5 & 4 & 4 & 5 & 5 & 4 & 4 & 5 & 5 & 4 & 5 & 4 & 5 & 5 & 4 & 77 \\
\hline $\mathrm{L}$ & 4 & 5 & 4 & 5 & 4 & 4 & 5 & 4 & 4 & 4 & 4 & 4 & 4 & 4 & 4 & 4 & 4 & 71 \\
\hline M & 4 & 4 & 4 & 5 & 5 & 4 & 4 & 5 & 5 & 4 & 5 & 5 & 4 & 5 & 4 & 5 & 5 & 77 \\
\hline $\mathrm{N}$ & 4 & 4 & 4 & 4 & 4 & 4 & 4 & 4 & 4 & 3 & 4 & 3 & 4 & 4 & 4 & 4 & 4 & 66 \\
\hline $\mathrm{P}$ & 3 & 3 & 3 & 3 & 3 & 4 & 3 & 3 & 3 & 4 & 3 & 3 & 3 & 4 & 4 & 3 & 3 & 55 \\
\hline $\mathrm{R}$ & 4 & 4 & 4 & 4 & 4 & 4 & 4 & 4 & 4 & 3 & 4 & 4 & 4 & 3 & 4 & 4 & 4 & 66 \\
\hline$S$ & 4 & 4 & 5 & 4 & 5 & 5 & 5 & 5 & 4 & 5 & 5 & 5 & 5 & 5 & 4 & 4 & 5 & 79 \\
\hline $\mathrm{T}$ & 4 & 4 & 4 & 4 & 3 & 3 & 4 & 4 & 4 & 3 & 3 & 4 & 4 & 4 & 4 & 4 & 4 & 64 \\
\hline$X$ & 5 & 4 & 5 & 4 & 4 & 4 & 4 & 4 & 4 & 5 & 4 & 5 & 5 & 5 & 5 & 5 & 5 & 77 \\
\hline Total & 50 & 50 & 50 & 50 & 50 & 50 & 50 & 50 & 50 & 50 & 50 & 50 & 50 & 50 & 50 & 50 & 50 & 850 \\
\hline
\end{tabular}

También se ha balanceado el grado de dificultad de cada lista y ordenado aleatoriamente los logatomos en cada una de ellas. La figura 5 muestra las curvas de mejor ajuste de cada lista en su correlación con el \% de articulación y STI, y comparadas con la curva de Houtgast y Steeneken.

Figura 5

Curvas lineales de mejor ajuste de las 17 listas en comparación a la curva de Houtgast y Steeneken.

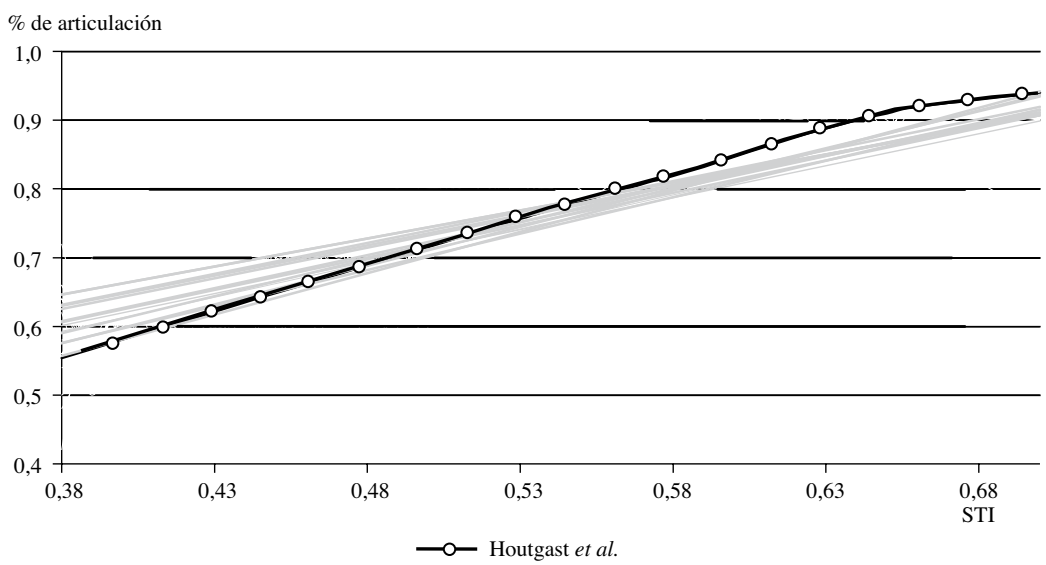


INTELIGIBILIDAD ACUSTICA EN ESPAÑOL: UNA PROPUESTA PARA SU MEDICION

Finalmente, la siguiente tabla muestra las 17 listas finales de 50 logatomos cada una.

Tabla 9

17 listas de 50 logatomos CVC fonéticamente balanceadas

\begin{tabular}{|c|c|c|c|c|c|c|c|c|c|c|c|c|c|c|c|c|}
\hline 1 & 2 & 3 & 4 & 5 & 0 & 7 & 0 & 9 & 10 & 11 & 12 & 13 & 14 & 15 & 16 & 17 \\
\hline JAR & FUK & REL & \begin{tabular}{|l} 
LUF \\
\end{tabular} & GUIN & CHAR & MIP & FICH & PUB & TUF & TAX & MICH & LAF & KEK & İUT & BUS & GLL \\
\hline RES & KAR & $\mathrm{CH}$ & GUIM & CHEJ & & DICH & $\mathrm{JOCH}$ & $\mathrm{OF}$ & SAX & & OM & PII & TIR & OM & UX & YIS \\
\hline TICH & LUD & GOL & $\mathrm{COCH}$ & $\mathrm{RUN}$ & B & DIM & GOJ & TAN & NIN & DEK & $10 \mathrm{~N}$ & & ก̃OJ & UR & MIJ & RUT \\
\hline JUD & SEP & FOS & cos & ÑAT & SACH & FOJ & PEJ & JIM & LOK & MII & RUCH & BUN & ROM & GUEK & TOCH & GAT \\
\hline MEX & LIT & GUET & СНECH & REX & SAI & GOCH & ÑOR & KOK & SUJ & BEN & SUK & $A B$ & $R$ & DIG & KACH & SICH \\
\hline SEN & NEX & PAS & SEK & RACH & KOS & TUG & YEX & SA & Po & SII & $J_{n}$ & & NAJ & CHEX & & WIJ \\
\hline DOL & SEB & JEJ & MIR & LUS & RAh & VER & LIN & $\mathrm{YU}$ & $\mathrm{KAB}$ & & $\mathrm{NOK}$ & LE & LECH & HOT & $\mathrm{EM}$ & NOT \\
\hline GUEN & FON & SIF & KUS & DOCH & NUF & GOK & CHAT & \begin{tabular}{|l} 
ÑUK \\
\end{tabular} & $\tilde{N} \mathrm{OS}$ & $\mathrm{RO}$ & $\tilde{\mathrm{N}} \mathrm{AB}$ & 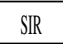 & LET & JUN & $\mathrm{PAJ}$ & $\overline{\mathrm{N} I X}$ \\
\hline DOG & FUM & ÑAM & LON & PAK & JAN & CHEL & CHEM & POR & FOT & LII & $\mathrm{YUJ}$ & MER & BAT & $B A X$ & ROJ & BOL \\
\hline PEM & RECH & YEN & JUF & LIB & LOL & NAX & YAM & BECH & CHEP & DAS & ROX & sos & RUX & OK & $A X$ & LAM \\
\hline FIT & LAN & KON & \begin{tabular}{|l|l|} 
NURR \\
\end{tabular} & BUG & SEX & GOB & CHII & LUP & NOM & NUX & MOJ & MUT & ÑOX & SIL & EB & KUR \\
\hline YOJ & TUT & LUL & RAS & TEK & $\mathrm{MU}$ & IIS & SAN & FIK & BEP & $\mathrm{PUCH}$ & FEK & BEL & $\mathrm{FEX}$ & $\mathrm{SOCH}$ & GUR & REN \\
\hline SER & YAJ & GUIK & $11 \mathrm{dJ}$ & BOJ & GUI & SAT & PAF & NOS & SON & MOK & DACH & DIIJ & GUN & YIN & JT & SUN \\
\hline $\mathrm{TAJ}$ & ROT & MIT & CHAL & $\mathbb{N P}$ & $\mathrm{BICH}$ & GUIR & $\tilde{N} \mathbb{P}$ & SUL & SOR & NOM & CHUS & KEM & NER & MET & NOT & DUF \\
\hline FOR & ÑOP & KUF & JUK & PAR & DIS & ÑEJ & NUK & YAP & KICH & FIN & MOR & IM & ROF & EK & MEM & DUS \\
\hline LAK & MAK & SEM & MAP & JOS & PAI & TAM & PAX & MAJ & KOF & FECH & JX & BAL & $\mathrm{ROCH}$ & GOP & YOF & CHUJ \\
\hline MAM & FOL & JUS & GUCl & NAP & No & SUR & YOB & $B I X$ & BEK & $\mathrm{KE}$ & $\mathrm{JOT}$ & $P O$ & NIK & MEP & VEL & $\mathrm{KAK}$ \\
\hline NELL & RIX & MAT & NAN & MAX & RUR & MOL & DER & СНОо & ÑUCH & LOX & JEM & $\dot{\mathrm{N} O \mathrm{~K}}$ & İIM & YUS & KAM & MIF \\
\hline GUF & $\mathrm{NACH}$ & NAK & PAC & MEJ & RIT & TACH & FIL & NUC & TUL & TOR & $\mathrm{N} \mathrm{NOCH}$ & TUK & KEL & CHIP & XIF & LEX \\
\hline $\mathrm{POT}$ & FOG & $\mathrm{KAX}$ & TEM & NUL & YIB & DOX & PON & NIR & RUM & CHIK & KES & GAK & DAL & $\mathrm{KEN}$ & Ñ̃ES & $\mathrm{NEL}$ \\
\hline ÑIJ & TIK & CHEF & YUT & YEM & $\mathrm{RC}$ & $J U X$ & TAK & PUN & KIM & $\mathrm{FE}$ & $\mathrm{NL}$ & PIJ & YOL & YIR & RIM & LUN \\
\hline PIX & NAM & $B U X$ & MUL & $\mathrm{JCH}$ & $\mathrm{FO}$ & ÑUJ & KIS & DAB & TAL & LICH & MIM & SUCH & BAN & MIX & M & BOX \\
\hline $\mathrm{DUK}$ & GOR & GUEB & SOP & BUP & MUS & BIK & $\mathrm{JOK}$ & \begin{tabular}{|l|l|}
$L U$ \\
\end{tabular} & MUP & CHUR & ÑIS & $\mathrm{NICH}$ & SUP & $\mathrm{JACH}$ & GAN & FAL \\
\hline SUT & JON & ĨEX & CHUF & PIT & $\mathrm{BO}$ & PAL & KUX & RUJ & TUB & POS & JET & MIK & MES & TOM & IIS & $\mathrm{YOCH}$ \\
\hline SIK & KAP & $\mathrm{RIJ}$ & $\mathrm{BIN}$ & YECH & $\sqrt{\mathbb{N}}$ & $\mathrm{KIN}$ & GOM & CHOM & JUT & YEJ & LIK & LAJ & CHUX & LEL & PER & $\mathrm{FAX}$ \\
\hline JAL & JAX & SIX & $\mathrm{Kl}$ & MON & $\tilde{\mathrm{N}} \mathrm{U}$ & PUL & $\mathrm{RL}$ & DOT & NUX & $\mathrm{CHE}$ & TUS & BUR & $S A B$ & FAS & TOK & YIG \\
\hline TIM & REM & YEP & NAT & BIS & TAS & DOF & GUIS & CHUM & KOX & CHOL & RIN & RUP & SOM & SUX & BOR & GUEM \\
\hline MOP & GUES & CHAK & LAT & TEL & $\mathrm{PC}$ & NUF & CHUL & RUF & KOJ & $\mathrm{K}$ & SOL & & RIS & DAN & OP & $\mathrm{YOK}$ \\
\hline MACH & $\mathrm{KUL}$ & $\mathrm{JOH}$ & JB & YIX & BII & MUK & SAF & KIL & JIK & 60 & TIP & KEP & FAK & JAM & BON & CHIS \\
\hline DCM & NUN & ÑUN & LEJ & DOK & $P$ & JOM & FIM & YAT & CHIJ & SLM & BIJ & MEN & DUR & JF & $\mathrm{RICH}$ & DON \\
\hline BOS & YICH & YER & $\mathrm{PO}]$ & RAM & KOF & SAK & KOM & LIS & CHUP & TON & JEP & FOCH & LEM & PICH & KEX & RIK \\
\hline PEB & YOX & TES & PAI & NAL & MUM & RAN & TA & GUT & GUECH & JES & DEL & LES & NUT & PIM & LAS & BAR \\
\hline$\dot{N} \mathrm{~N} F$ & CHACH & LAP & ÑUM & KUK & DON & TEX & KUP & ÑOL & $\mathrm{PU}$ & NEF & BAM & EX & LEP & POX & IIX & YUX \\
\hline KAT & GUICH & GOG & SEL & YIF & $\mathrm{NES}$ & CHAS & BES & $\operatorname{RAX}$ & GUIX & PIR & LER & NOX & $\mathrm{POK}$ & SEJ & $\mathrm{TLL}$ & LOP \\
\hline REP & SES & JUJ & DIP & LUM & LUK & CHUK & $\mathrm{GOF}$ & RON & BAS & $\mathrm{BACH}$ & RIR & YIP & BOP & NOR & GUEL & PEP \\
\hline MUN & PIJ & MAN & NIT & MUF & NEJ & TUN & СНОСН & IICH & DEM & FET & YOR & KOT & $\mathrm{RIP}$ & SOF & FUL & TOJ \\
\hline ÑEK & PEL & NUR & NAR & PUR & RAJ & NUM & RAL & LOCH & LIL & $\mathrm{NOCH}$ & TUM & SIN & CHIN & FEL & CHIR & LACH \\
\hline PECH & TIF & ÑACH & TUR & FUX & YEK & DUT & DIR & FER & MIS & FAM & BEX & KET & ĨICH & TUP & FAT & NEB \\
\hline NAS & NAS & YIK & TIX & $\mathrm{DAF}$ & $\mathrm{GU}$ & RAP & BOT & DES & YAN & YUL & JECH & PUX & CHOS & YOS & СHOX & BLM \\
\hline $\mathrm{BUCH}$ & REK & SECH & DUX & SAS & BU] & $\tilde{\mathrm{N}} \mathrm{AF}$ & PUJ & $\mathrm{CHICH}$ & DIX & LOJ & NOF & KECH & $\mathrm{JIJ}$ & LIJ & MEK & BAP \\
\hline
\end{tabular}




\begin{tabular}{|c|c|c|c|c|c|c|c|c|c|c|c|c|c|c|c|c|}
\hline 1 & 2 & 3 & 4 & 5 & 6 & 7 & 8 & 9 & 10 & 11 & 12 & 13 & 14 & 15 & 16 & 17 \\
\hline DAP & PIF & BOCH & ÑA & KOL & ÑAL & DIL & FOX & YAK & LEF & NUP & LUT & CHOR & JAS & YUCH & PET & NECH \\
\hline GAX & PEK & GUK & GUEX & CHAM & PAN & LOS & DIK & CHEN & MOCH & KUT & ÑU & CHUT & TAG & NER & YUP & DOR \\
\hline CHIX & YIM & POP & BOK & LUJ & SOX & LUCH & FUT & JOX & RUS & DEP & JEN & YUM & GAM & NIK & KUN & RER \\
\hline JUL & DOS & LAX & MAL & NAN & NAX & GUP & JIR & GUT & JER & BAG & ÑU & DEX & TOS & BUL & NECH & PES \\
\hline KAN & TOT & BOM & PEX & CHUCH & FUP & MOT & YACH & PIP & TEJ & TUJ & CHL & DIF & GACH & TAB & DAD & YET \\
\hline TOX & TER & SOT & JUM & FOM & LIP & YAS & TEN & BEM & DECH & NIS & MOX & CHIM & FF & FACH & NEP & MAS \\
\hline CHAF & MUR & GUS & TECH & FR & MECH & NUS & MUCH & JEF & BET & LAB & GAG & TOL & LOT & POJ & PEN & SIM \\
\hline DUCH & KAL & LAL & PUG & LOR & DOJ & SIT & PUF & JOJ & CHOK & BAJ & YEF & KIX & TUCH & BAK & YUK & LED \\
\hline LUR & NOL & MAR & JOL & YOT & CHEK & NON & MOS & MIN & ÑR & LOF & CHET & KAS & LIX & SIS & JAK & NEK \\
\hline FUS & BUJ & GUM & YES & SUS & SAR & KIR & ÑS & PUS & FES & RUK & ROS & GAS & TIN & DAT & PIK & NEM \\
\hline
\end{tabular}

\section{BALANCE}

En este trabajo se ha aplicado la medición de la inteligibilidad con empleo de un corpus inicial adecuado al español panhispánico de 1200 logatomos que representan de manera exhaustiva las combinaciones posibles de estructura Consonante + Vocal + Consonante existentes en él. Los resultados de estas mediciones han sido pareados con los resultados de las mediciones de STI, junto con los porcentajes de medición subjetiva del inglés según las listas establecidas por Houtgast y Steeneken. Lo anterior ha permitido proponer un nuevo corpus para evaluar la inteligibilidad del español de 850 logatomos que conserva la estructura CVC, clasificados en 17 listas fonéticamente balanceadas que tienen en cuenta, de acuerdo a lo constatado en este mismo trabajo, especialmente la importancia de la segunda consonante en el grado de efectividad de los resultados.

Finalmente, con el objeto de afinar esta propuesta y hacerla funcionalmente equivalente al STI para la medición acústica de los recintos, en español, se ha empezado a trabajar en la actualidad, en tareas de aplicación y evaluación del corpus diseñado.

\section{OBRAS CITADAS}

Egan, FP. 1948. “Articulation testing methods". Laryngoscope 58: 955-991.

Gavilán, J. 1988. Fonoaudiología para educadores. Madrid: Universidad Nacional de Educación a Distancia.

Houtgast, T. y J. M. Steeneken. 1973. "The Modulation Transfer Function in room acoustics as a predictor of speech intelligibility". Acustica, 28 (1): 66-73.

-1985. "The Modulation Transfer Function MDT in room acoustics". $B \& K$ Tech. Rev., 3, 3-29.

Llisterri, J. 1991. Introducción a la fonética: el método experimental. Barcelona: Editorial Anthropos.

Peutz, V.M.A. 1971. "Articulation loss of consonants as a criterion for speech transmission in a room", Journal of Audio Engineering Society. V. 19, No 11: 23-27.

Sommerhoff, J. y C. Rosas. 2007. Evaluación de la inteligibilidad del habla. Estudios Filológicos 42: 215-225.

—. (en preparación). “Existe la inteligibilidad absoluta? fonemas críticos en español”. 